\title{
ASCO 2021 - selection of personal highlights in early stage non-small cell lung cancer
}

\author{
Gudrun Absenger · Andreas Pircher (D)
}

Received: 9 September 2021 / Accepted: 4 October 2021 / Published online: 18 October 2021

(C) The Author(s) 2021

\begin{abstract}
Summary This article intends to summarize personal non-small cell lung cancer (NSCLC) highlights of the virtual ASCO 2021 meeting. Immunotherapy is now a mainstay of advanced stage NSCLC treatment and there are several ongoing studies investigating the role of immunotherapy in early stage NSCLC. At ASCO 2021 the first data on atezolizumab in the adjuvant setting were presented and give a positive signal that immunotherapy will also become an option for patient in early stage NSCLC. Furthermore, overall survival (OS) updates of two studies investigating the effects of epidermal growth factor receptor (EGFR) tyrosine kinase inhibitors (TKIs) in the adjuvant setting of EGFR-mutated NSCLC patients were presented. In conclusion ASCO 2021 provided the lung cancer community with inspiring new data especial in early stages and challenges the community with integration of these data into our daily clinical routine.
\end{abstract}

Keywords ASCO meeting 2021 - NSCLC · EGFR · Adjuvant immunotherapy · Atezolizumab

\section{Introduction}

The virtual ASCO 2021 took place on June 4-8, 2021 and treatment of non-small cell lung cancer (NSCLC) was again a major aspect of the meeting. The congress

\section{G. Absenger}

Division of Oncology, Department of Internal Medicine,

Medical University of Graz, Auenbruggerplatz 15, 8036 Graz, Austria

\section{PD Dr. A. Pircher $(\bowtie)$}

Department of Hematology and Oncology, Internal

Medicine V, Medical University of Innsbruck,

Anichstraße 35, 6020 Innsbruck, Austria

andreas.pircher@i-med.ac.at was held under the tagline "equity: every patient, every day and everywhere".

In regard to NSCLC the use of immunotherapy in early stage disease was of major focus and the first data of atezolizumab in the adjuvant setting were presented. Furthermore, updates on studies investigating the effects of EGFR TKIs in the adjuvant setting of EGFR-mutated NSCLC patients were presented. In advanced stage disease interesting however not yet practice changing abstracts were discussed. Therefore, this personal highlight article focuses on the topics on adjuvant immunotherapy and adjuvant EGFR TKI therapy in EGFR-mutated patients.

\section{Early stage non-small cell lung cancer}

\section{Immunotherapy as adjuvant therapy}

\section{IMpower 010}

Heather Wakelee presented the first results of IMpower 010, a phase III, multicentre, open-label, randomized study. The trial enrolled patients with completely resected stage IB to IIIA NSCLC according to the UICC 7 staging and ECOG 0-1 who had received up to four cycles of cisplatin-based chemotherapy with pemetrexed, docetaxel, gemcitabine or vinorelbine. A total of 1005 patients were randomized 1:1 to 16 cycles of atezolizumab $1200 \mathrm{mg}$ every 3 weeks vs best supportive care (BSC). The primary endpoint of investigator assessed disease-free survival (DFS) and the secondary endpoint of overall survival (OS) were tested hierarchically starting with DFS in PD-L1 TC $\geq 1 \%$ in stage II-IIIA, then DFS in all stage II-IIIA patients and finally DFS in an intention to treat (ITT) population.

At ASCO 2021 the first results from the preplanned interim analysis at a median follow-up time of 32.2 months were presented. Atezolizumab showed signif- 
icant benefit DFS in PD-L1 positive (PD-L1 TC $\geq 1 \%$ ) in the stage II-IIIA patients. Median DFS was not reached in the atezolizumab arm vs. 35.3 months with BSC (HR 0.66; 95\% CI 0.50-0.88, $p=0.0039$ ) [1].

In all stage II-IIIA patients regardless of PD-L1 status the benefit was also significant but lower: 42.3 months with atezolizumab vs. 35.3 months with BSC (HR 0.79; 95\% CI 0.64-0.96, $p=0.02$ ) [1]. In the subgroup analysis mainly the PD-L1 positive ( $\mathrm{TC} \geq 1 \%$ ) and high positive (TC $>50 \%$ ) patients benefit from atezolizumab; in contrast the PD-L1 negative patients had no benefit. In addition, no benefit was seen for EGFR- and ALK-positive patients. The significance boundary was not crossed for DFS in the ITT population, the OS data was immature and not formally tested.

Adverse advents (AEs) of any grade occurred in $92.7 \%$ of patients receiving atezolizumab and $70.7 \%$ of BSC patients; grade 3/4 events in 21.8 and $11.5 \%$ respectively. Treatment was discontinued due to AEs in $18.2 \%$ of atezolizumab patients [1].

Heather Wakelee concluded that atezolizumab may be considered a practice changing adjuvant treatment option for patients with stage II-IIIA PD-L1 positive NSCLC. Nevertheless, we need to wait for further results.

\section{Targeted therapy in genetic driven early stage NSCLC}

In EGFR-mutated metastatic NSCLC, frontline EGFR inhibition is the gold standard and three different generations of EGFR inhibitors are approved. At the ASCO 2021 two relevant trials evaluating TKIs in early stage EGFR-mutated NSCLC were presented.

The phase III IMPACT trial evaluated adjuvant gefitinib vs. cisplatin/vinorelbine in Japanese patients with completely resected EGFR-mutated stage II-III NSCLC. All patients had common EGFR mutations, either deletion 19 or L858R and median duration of follow-up was 71 months. The primary endpoint DFS was 36 months with gefitinib versus 25 months with chemotherapy. However, after 5 years, the Kaplan-Meier curves began to overlap and no significant difference in DFS was seen (HR 0.92; 95\% CI 0.67-1.28; $p=0.63$ ) [2]. The DFS rates at 5 years were $31.8 \%$ with gefitinib and $34.1 \%$ with chemotherapy. OS was also similar in each arm, the 5-year survival rates for gefitinib and cis/vin arms were 78.0 and $74.6 \%$, respectively (HR for death of 1.03 ; 95\%CI, 0.65-1.65; $p=0.89$ ) [2].

It can be concluded that adjuvant gefitinib may prevent early relapse, but did not significantly prolong DFS or OS in patients with completely resected stage II-III, EGFR-mutated NSCLC.

Further relevant data concerning neoadjuvant/ adjuvant TKI treatment at ASCO 2021 the final OS analysis of phase II CTONG1103 trial were presented. In all, 72 Chinese patients with stage IIIA N2 NSCLC and common EGFR mutations received either neoadjuvant erlotinib $150 \mathrm{mg}$ /day for 42 days and as adjuvant therapy, up to 12 months or gemcitabine plus cisplatin two cycles neoadjuvant and up to two cycles adjuvant. The progression-free survival (PFS) data were presented previously and showed a significant benefit for the TKI versus chemotherapy. Median PFS with erlotinib was 21.5 months versus 11.4 months with chemotherapy (HR 0.39 ; 95\% CI 0.23 to 0.67 ; $p<0.001$ ) [3]. For OS, the median follow-up was 62.5 months and found no significant difference. OS was 42.2 months with erlotinib and 36.9 months with chemotherapy (HR 0.83, 95\%CI 0.47-1.47, $p=0.513$ ). The 3- and 5-year OS rates were 58.6 and $40.8 \%$ in the erlotinib arm and 55.9 and $27.6 \%$ with chemotherapy, respectively [4].

\section{Conclusion}

IMpower 010 is the first study showing a DFS benefit of atezolizumab compared to BSC in early stage NSCLC. The study bears practice changing potential, nevertheless several open questions have to be addressed before regulatory approval. The benefit of atezolizumab is mainly seen in PD-L1 TPS high patients and less in PD-L1 $>1 \%$ and even absent in negative patients. Therefore, patient selection will be key especially in light of the potential high-grade immune-related adverse events (irAE) reported in the study. The OS endpoint is not mature yet; however the curve shape of both treatment groups seems to separate nicely and interpretation of this consistent split of the curves may suggest that immunotherapy cures patients in early stages and recapitulates the efficacy from advanced stage studies. Nevertheless, OS data are of major importance as both mentioned studies with EGFR TKI in early stage NSCLC neo-/adjuvant setting were positive in DFS/PFS; however this effect did not translate into an OS benefit. One explanation could be that it is unusual for a targeted therapy to actually cure lung cancer as remissions are rarely sustained when patients stop treatment. This could be one difference in the discussion of adjuvant therapy with targeted therapies compared to immunotherapy. And it raises the question whether the recent approval of adjuvant osimertinib based on the impressive DFS benefit of the ADAURA trial will help us to cure more EGFR-positive lung cancer patients.

There are still several open considerations for the optimal endpoint in neo/adjuvant NSCLC therapy which give room for lively discussion at the next upcoming meetings.

Funding Open access funding provided by University of Innsbruck and Medical University of Innsbruck.

Conflict of interest G. Absenger has received honoraria for advisory boards from: AMGEN, AstraZeneca, BMS, Böhringer Ingelheim, Lilly, MSD, Novartis, Pfizer, Roche, Sanofi, Takeda. A. Pircher has received speaker's fee and honoraria for advisory 
boards from: Astra Zeneca, BMS, Böhringer Ingelheim, Roche, Pfizer, Takeda and MSD.

Open Access This article is licensed under a Creative Commons Attribution 4.0 International License, which permits use, sharing, adaptation, distribution and reproduction in any medium or format, as long as you give appropriate credit to the original author(s) and the source, provide a link to the Creative Commons licence, and indicate if changes were made. The images or other third party material in this article are included in the article's Creative Commons licence, unless indicated otherwise in a credit line to the material. If material is not included in the article's Creative Commons licence and your intended use is not permitted by statutory regulation or exceeds the permitted use, you will need to obtain permission directly from the copyright holder. To view a copy of this licence, visit http://creativecommons.org/licenses/by/4.0/.

\section{References}

1. Wakelee HA, et al. IMpower010: Primary results of a phase III global study of atezolizumab versus best supportive care after adjuvant chemotherapy in resected stage IBIIIA non-small cell lung cancer (NSCLC). J Clin Oncol. 2021;39(15_suppl):8500-8500. https://doi.org/10.1200/ JCO.2021.39.15_suppl.8500.

2. Tada $\mathrm{H}$, et al. Adjuvant gefitinib versus cisplatin/ vinorelbine in Japanese patients with completely resected, EGFR-mutated, stage II-III non-small cell lung cancer
(IMPACT, WJOG6410L): A randomized phase 3 trial. J Clin Oncol. 2021;39(15_suppl):8501-8501. https://doi.org/10. 1200/JCO.2021.39.15_suppl.8501.

3. ZhongWZ, et al. Erlotinib versus gemcitabine plus cisplatin as neoadjuvant treatment of stage IIIA-N2 EGFR-mutant non-small-cell lung cancer (EMERGING-CTONG 1103): a randomized phase II study. J Clin Oncol. 2019;37:2235-45. https://doi.org/10.1200/JCO.19.00075.

4. Wu YL, et al. CTONG1103: final overall survival analysis of the randomized phase 2 trial of erlotinib versus gemcitabine plus cisplatin as neoadjuvant treatment of stage IIIA-N2 EGFR-mutant non-small cell lung cancer. J Clin Oncol. 2021;39(15_suppl):8502-8502. https://doi.org/10. 1200/JCO.2021.39.15_suppl.8502.

Publisher's Note Springer Nature remains neutral with regard to jurisdictional claims in published maps and institutional affiliations.

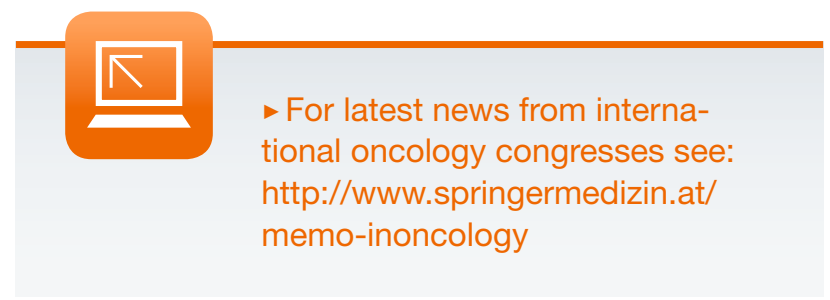

